\title{
Crystal Growth of IRMOF-3 (Isoreticular Metal-Organic Frameworks-3) Synthesized using Solvothermal Method
}

\author{
Pemta Tia Deka ${ }^{1}$, Ratna Ediati ${ }^{1}$, and Didik Prasetyoko ${ }^{1}$
}

\begin{abstract}
IRMOF-3 crystals have been synthesized using solvothermal method by reacting zinc nitrate hexahydrate with 2amino-1,4-benzenedicarboxylic acid in $N^{\prime} N$-dimethylformamide ( DMF ) at $100{ }^{\circ} \mathrm{C}$ for $18,24,48,72,120$ and $144 \mathrm{~h}$ to observe crystal growth. The obtained crystals were characterized using $X$ - ray Diffraction (XRD), SEM ( Scanning Electron Microscopy) and Scanning Electron Microscopy - Energy Dispersive X-ray ( SEM - EDX ). Diffractograms of crystals synthesized at heating times of 24 and 48 hours showed major peaks at $2 \theta$ of 6.8; 9.6 and 13.7, which are the characteristic peaks of an IRMOF-3 crystal. The size of IRMOF-3 crystals observed using SEM increased with the increase in heating time up to $48 \mathrm{~h}$, then decreased at heating times of 72, 120 and $144 \mathrm{~h}$. The largest IRMOF-3 crystal is of cube shape with a particle size of $500 \mu \mathrm{m}$. The weight of the resulting crystals increased with the increase in heating time, then remained constant after $72 \mathrm{~h}$.
\end{abstract}

Keywords — crystal growth, IRMOF-3, solvothermal.

\section{INTRODUCTION}

$\mathrm{M}$ etal organic framework (MOF) is a porous material that attracted the attention of many researchers associated with the structure and pore size can be set. This is due to a combination of organic ligands and different metal on the MOF will form crystalline structures vary, the variations are endless geometry structure, variation of pore size and generate MOF with high crystallinity

IRMOF-3 atau Isoreticular Metal Organic Framework3 is one type of MOF consisting of metal ions $\mathrm{Zn}$ as the central atom and the organic ligand benzene dicarboxylic (BDC) which has a group-NH2 as a linker in the framework formation. The presence of $\mathrm{N}$ atoms in IRMOF-3 can act as active centers of the hydrogen thus increasing the energy storage properties associated with its application as a hydrogen storage material [10]. In addition, the amino group of the organic ligand IRMOF3 can act as the active site of the catalyst, for example a Pall-Knorr reaction [8].

Several methods have been used for the synthesis of various types of MOF include solvothermal, sonochemical, microwave radiation, and mechanochemical. However, most of the MOF synthesized by reaction solvothermal namely by reacting organic ligands and metal salts at low temperature is below $300 \mathrm{oC}$ [6]. Research on synthesis of IRMOF-3 has been carried out by Rowsell et al., (2006) solvothermal method by reacting $\mathrm{Zn}(\mathrm{NO} 3) 2.4 \mathrm{H} 2 \mathrm{O}$ and 2-amino-1, 4 benzenedicarbocylic acid in Diethylformamide (DEF) media is heated at $100{ }^{\circ} \mathrm{C}$ for 18 hours. IRMOF- 3 crystals synthesized has a surface area of $2446 \mathrm{~m} 2 / \mathrm{g}$ and a total pore volume of $1.07 \mathrm{~cm} 3$ / g. Then Britt et al., (2008) also perform synthesis of IRMOF-3 with the same method by Rowsell et al., (2006), but used the old heating is 15 hours. The surface

${ }^{1}$ Pemta Tia Deka, Ratna Ediati, and Didik Prasetyoko are with Departement of Chemistry, Faculty of Mathematical and Science Institut Teknologi Sepuluh Nopember, Surabaya, 60111, Indonesia.

E-mail: Tia.deka12@mhs.chem.its.ac.id; rediati@chem.its.ac.id; didikp@chem.its.ac.id. area and pore volume of the resulting row is $1568 \mathrm{~m} 2 / \mathrm{g}$ and $1.07 \mathrm{~cm} 3 / \mathrm{g}$ [1].

Several other researchers have done a synthesis of IRMOF-3 using the reactants and heating temperature are the same as Rowsell and Britt, but the solvent is dimethylformamide (DMF) with varying heating times which is 18 hours, 24, and 48 hours $[2,3,5,8,12]$. The surface area of IRMOF-3 were produced between 2351 and $2446 \mathrm{~m} 2 / \mathrm{g}$ with a pore volume of between 0.9 and $1.02 \mathrm{~cm} 3 / \mathrm{g}$. Effect of prolonged heating in the synthesis of IRMOF-3 with solvetermal method on the characteristics and growth of crystals that form has not been reported.

Based on the description above, this research was conducted on the synthesis of IRMOF-3 solvothermal method by reacting $\mathrm{Zn}$ (NO3) $2.6 \mathrm{H} 2 \mathrm{O}$ and 2 -amino-1, 4benzenedicarboxylic acid in DMF solvent then heated at $100^{\circ} \mathrm{C}$ for the time variation of $18,24,48,72,120$, and 144 hours to determine the effect of prolonged heating on the characteristics and growth of crystals of IRMOF-3 synthesis results.

\section{METHOD}

\section{A. Synthesis of IRMOF-3}

IRMOF-3 crystals synthesized using solvothermal method by reacting $0.9683 \mathrm{~g} \mathrm{Zn}(\mathrm{NO} 3) .4 \mathrm{H} 2 \mathrm{O}$ (SigmaAldrich, $99.0 \%), \quad 0.2293 \mathrm{~g}$ of 2-amino-1,4benzenedicarbocylic acid (Sigma-Aldrich , 99.0\%), and $30 \mathrm{~mL}$ of solvent $\mathrm{N}$ 'N-dimethyl formamide (DMF, Merck, 99.8\%) in a sealed reaction bottle. After that, the mixture was heated at $100^{\circ} \mathrm{C}$ for $18,24,48,72,120$, and 144 hours. The next stage is followed by cooling and decantation process. Filtrate mixture into the bottle vial accommodated while solid results during the decantation washed once a day using $30 \mathrm{~mL}$ DMF. Subsequently, 50 $\mathrm{mL}$ of chloroform (Merck, 99.9\%) was added to the above solution and the mixture allowed to remain joined crystal solvent for almost 1 day. The next stage is done the mixture decantation, and crystalline solids were washed again using chloroform once daily for 3 days.

In the last crystal leaching, solvent chloroform was removed in vacuum for 3-4 hours. IRMOF-3 solids are 
stored in small vials bottles in a nitrogen atmosphere (Sumida dkk., 2010).

\section{B. Characterization}

XRD patterns of the samples obtained from the XRDJEOL using $\mathrm{Cu} \mathrm{K} \alpha$ radiation $(1=1.5405 \AA$ ) at $40 \mathrm{kV}$ and $30 \mathrm{~mA}$, and recorded on a scale of $5-50^{\circ} 2 \theta$, a scan speed of $0.04^{\circ} / \mathrm{sec}$. Data obtained in the form of price $2 \theta$, d spacing and intensity of the diffraction peaks. Zeiss Scanning Electron Microscopy (EVO MA10) equipped with Energy Dispersive X-ray (SEM-EDX) was used to determine the morphology and elemental content of the surface of the product.

\section{RESULT AND DISCUSSION}

\section{A. Synthesis Crystal IRMOF-3}

The whole crystal IRMOF-3 synthesis results are fawn colored. The crystals stick to the walls and bottom of the bottle reaction. The resulting filtrate was clear yellow colored. In the heating time of 18 to 48 hours, the resulting filtrate thinner and began clear back in time 72 to 144 hours. This is possible because when the crystal was heating at 48 hours, many ligands and the metal can react with DMF solvent so that the resulting filtrate concentrated. In addition, if the filtrate to 48 hours left at room temperature, a yellowish brown precipitate appeared. This suggests that crystal growth can still continue, but it requires a very long time that is up to 1 month.

When the solvent DMF was replaced with chloroform, the resulting filtrate was clear colored. Crystal IRMOF-3 at heating time 18 hours having a bit detached from wall when washing with chloroform. In contrast, crystal IRMOF-3 at other heating time are still stuck firmly in the walls and bottom of the bottle. IRMOF-3 crystals synthesized along with the filtrate and crystal IRMOF-3 after vacuum successive shown by Figures 1 and 2 .

Based on the results of weighing the crystal IRMOF-3, crystals weight growing along with the increasing synthesis time. In the crystal IRMOF-3 (72, 120 and 144) hours, crystal constant weight was obtained. This is because the rising time of the synthesis, the crystal growth of IRMOF-3 went slower. This can be seen by the weight did not change significantly. The results of IRMOF-3 crystals weighing from a variety of heating time at $100^{\circ} \mathrm{C}$ is shown in Figure 3 and Table 1.

\section{B. Results Characterization of IRMOF-3 Using XRD}

Characterization using XRD aims to determine the crystallinity of synthesized IRMOF-3. $2 \Theta$ angle used is 5-50o. The resulting XRD pattern of the crystal IRMOF324 and 48 h showed the characteristic peak at $2 \Theta(6.80$, 9.60 and 13.7o). The pattern is suitable with the simulated diffractogram IRMOF-3 (Yim et al., 2012).

Diffractogram crystals during 18 hours showed that the crystal IRMOF-3 has begun to form and characterized by the appearance of characteristic peaks of IRMOF-3. However, another peak appeared at $15.352 \Theta 19.39$, and 24.62. The crystallinity of IRMOF-3 $18 \mathrm{~h}$ is still small compared with other IRMOF-3 because there are other phases are formed in those crystal. Furthermore, IRMOF-3 that produces the highest crystallinity is the heating time of 72 hours. At the time 120 and 144 hours, the crystallinity is almost same but start to decline compared with 72 hours. This is possible because the crystal growth IRMOF-3 is no longer happened. The weak intensity at longer time solvothermal indicate other compounds that are formed from metal oxides, for example $\mathrm{Zn}$ is $\mathrm{Zn}(\mathrm{OH}) 2$. The results of XRD crystallinity of IRMOF-3 for a wide variety of heating time in a row as shown in Table 2 and Figure 4.

\section{Results Characterization Using Scanning Electron Microscopy (SEM) and Scanning Electron Microscopy-Energy Dispersive X-ray (SEM-EDX)}

Characterization using SEM aims to determine the surface morphology of the crystal IRMOF-3. SEMEDX, while it can be used to determine the elemental information on the particle surface. Based on the results of SEM characterization showed that the crystal IRMOF-3 from the 18, 24, 48, 72, 120, and 144 hours has a cubic structure with the largest particle size of 500 $\mathrm{lm}$. Based on physical observation, the largest size of the crystals synthesized IRMOF-3 is located on the heating time of 48 hours, while the 120 and 144 hours crystal size is smaller than the overall crystal synthesis results. In addition, the surface of the crystal IRMOF-3 ranging from 72 to 144 hours looks rough and there are cracks and not uniform in size, whereas the surface of IRMOF-3 18, 48 and 24 hours looks smooth and uniform in size. This is possible because the longer the time solvothermal the IRMOF-3 crystals growing increasingly imperfect

SEM results are consistent with studies that have been conducted by Phan et al., (2012) on the synthesis of IRMOF-3 is solvothermal which would then be applied as a catalyst for the reaction of Pall-Knor. IRMOF-3 crystal form produced is a cube with crystal size between 400 and $500 \mathrm{~nm}$.

\section{Results Characterization Using Scanning Electron Microscopy-Energy Dispersive X-ray (SEM-EDX)}

Characterization using SEM-EDX aims to determine the percent of elements in the surface of the material synthesized khususya nitrogen atoms of IRMOF-3. In this study, crystal IRMOF-3 SEM-EDX characterization was 18,72 and 48 hours for 18 hours the crystals have a smooth surface, then the highest crystallinity lies in the crystal while the crystal 72 hours to 48 hours to have the largest crystal size.

The results of SEM-EDX characterization showed that the longer the time solvothermal, the amount of nitrogen contained also diminishing. In crystals 18,48 and 72 hours the amount of nitrogen obtained successively, namely $6.62 ; 6.45$ and $5.42 \mathrm{wt} \%$. The longer the time solvothermal, the more organic ligands that react with $\mathrm{Zn}$ metal. Therefore, the nitrogen atoms contained in the organic ligands will be experiencing a decrease in number with increasing heating time.

Based on the above characterization results, increasing $\mathrm{Zn}$ levels rose from 48 to 72 hours. Analysis of the element in the crystal IRMOF-3 was also carried out by Rowsell et al., (2005) with the following results: C 35.4 / 35.5; H 1.86 / 2.00, N 5.16 / 5:39; Zn 32.1/28.8. The amount of nitrogen obtained almost the same as the research gasil, whereas $\mathrm{Zn}$ levels obtained in this study is less. This is possible because a lot of $\mathrm{Zn}$ metal reacting with organic ligan. However, the crystals obtained 48 hours more nitrogen than the others. It show that 
nitrogen is not much decomposed at current synthesis processes.

\section{CONCLUSION}

Synthesis of IRMOF-3 has been successfully carried out using methods solvothermal in DMF solvent at the optimum time of heating 24 hours at a temperature of $100^{\circ} \mathrm{C}$. Based on the results of characterization using XRD, SEM, and SEM-EDX $2 \Theta$ characteristics of the obtained peak $\left(6.8^{\circ} ; 9.6^{\circ} ; 13.7^{\circ}\right)$. Furthermore, crystals synthesized IRMOF-3 has a shape of a cube with a crystal size of $500 \mathrm{~nm}$ at heating time $24 \mathrm{~h}$. Based on the results of SEM-EDX showed that the longer the heating time, the surface of IRMOF-3 crystal look more rugged and the amount of nitrogen also becomes smaller.

\section{ACKNOWLEDGEMENT}

This research was supported by the Directorate General of Higher Education (Directorate General of Higher Education) and chemical research laboratory, Institute of Technology Surabaya November.

\section{REFERENCES}

[1]. Britt, D., Tranchemontagne, D., dan Yaghi, O., "Metal Organic Frameworks With High Capacity and Selectivity for Harmful Gases", Proceedings of The National Academy of Sciences, 2008, Vol.105, pp. 11623-11627.

[2]. Britt, D., Lee, C., Uribe-Romo, F., Furukawa, H., dan Yaghi, O.M., "Ring-Opening Reactions Within Porous Metal-Organic Frameworks", Inorganic Chemistry Communications, 2010 , Vol.49, pp. 6387-6389.

[3]. Cao, W., Li, Y., Wang L., dan Liao S., "Effects of Metal Ions and Ligand Functionalization on Hydrogen Storage in Metal Organic
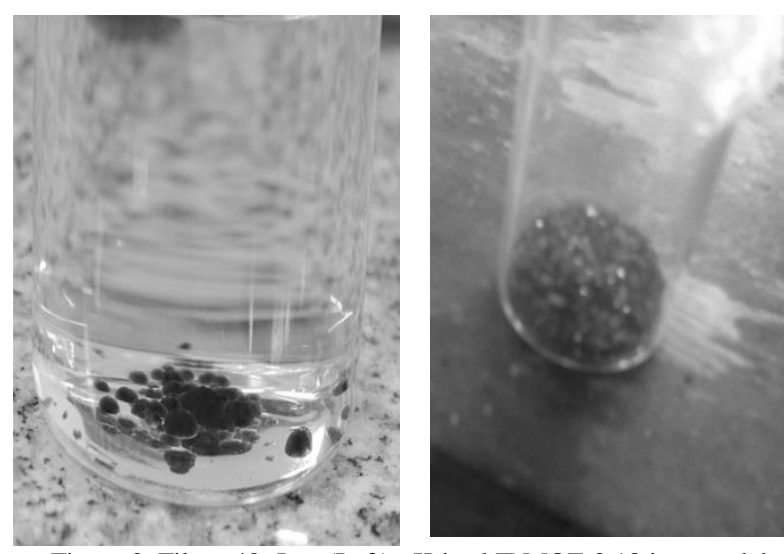

Figure 3. Filtrat 48 Jam (Left); Kristal IRMOF-3 18 jam setelah vakum (right)

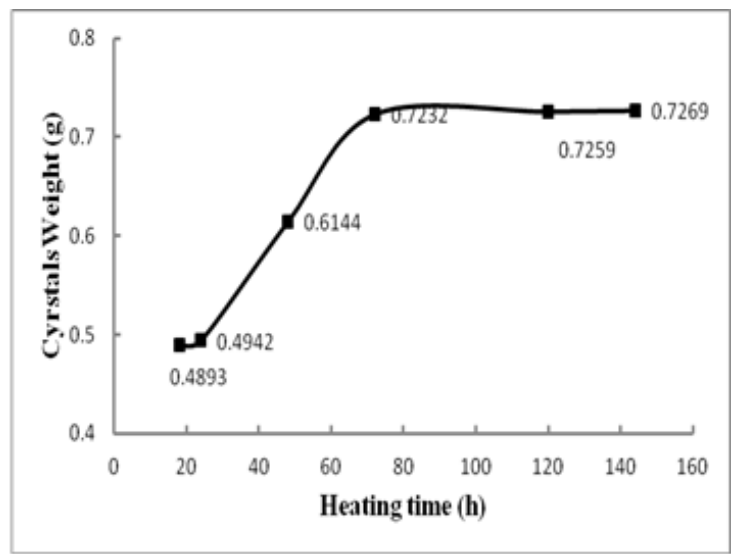

Figure 2. Crystal IRMOF-3 weight at various heating time
Frameworks by Spillover". Journal of American Chemical Society, 2011, Vol. 115, pp.13829-13836.

[4]. Chen, M., Yang, Y., Zhang, C., Kuang, D., Deng, Y., dan Chen, Z., "Solvothermal Synthesis, Structure and Properties of a New 3D Anionic Porous Framework", Inorganic Chemistry Communications, 2011, Vol. 14, pp. 444 - 446.

[5]. Gascon, J.,Aktay U., Maria D., Gerard P., Kapteijn F., , “Amino Based Metal-Organic Frameworks as Stable, Highly Active Basic Catalysts", Journal of Catalysis, 2009, Vol.261, pp.75-87.

[6]. Kuppler, R.J., Timmonsb, D.J., Fanga, Q.R., Lia,J.R., Makala, T.A., Young, M.D., Yuan, D., Zhao, D., Zhuang, W., Zhou, H.C., "Potential Applications of Metal Organic Frameworks", Coordination Chemistry Reviews, 2009, Vol. 253,pp. 3042-3066.

[7]. Natarajan, S., Mahata, P., dan Sarma, D., (2012), "The Relevance of Metal Organic Frameworks (MOFs) in Inorganic Materials Chemistry", Journal of Chemical Science, 124, 339-353

[8]. Phan, N.T.S , Nguyen, T.T., Luu, Q.H., Nguyen, L., "Paal-Knorr Reaction Catalyzed by Metal Organic Framework IRMOF-3 as an Efficien and Reusable Heterogeneous Catalyst", Journal of Molecular Catalys, 2012, Vol. 363 - 364, pp. $178-185$.

[9]. Rowsell, J. dan Yaghi, O.M., , "Effects of Functionalization, Catenation, and Variation of the Metal Oxide and Organic Linking Units on the Low Pressure Hydrogen Adsorption Properties of Metal-Organic Frameworks", Journal of American Chemical Society, 2005, Vol. 128, pp. 1304-1315.

[10]. Sankaran, M. dan Viswanathan, B., "Nitrogen-Containing Carbon Nanotubes as a Possible Hydrogen Storage Medium", Indian Journal of Chemistry, 2001, Vol.47A, 808-814.

[11]. Sumida, K., dan Arnold, J.,"Preparation, Characterization, and Postsynthetic Modification of Metal-Organic Frameworks:Synthetic Experiments for an Undergraduate Laboratory Course in Inorganic Chemistry", Journal of Chemical Education, 2011, Vol. 88,pp. 92-94.

[12]. Yang, Seung., Kim, T., Im, J.H., Kim, Y.S., Lee, K., Jung, H., dan Park, C.R., , "MOF-Derived Hierarchically Porous Carbon with Exceptional Porosity and Hydrogen Storage Capacity", Journal of American Chemical Society, 2012, Vol.24, pp. 464-470.

[13]. Zhao, D., Yuan, D., dan Zhou, G.,, "The Current Status of Hydrogen Storage in Metal Organic Frameworks", Energy \& Environmental Science, 2008, Vol. 1, pp. 222-235.

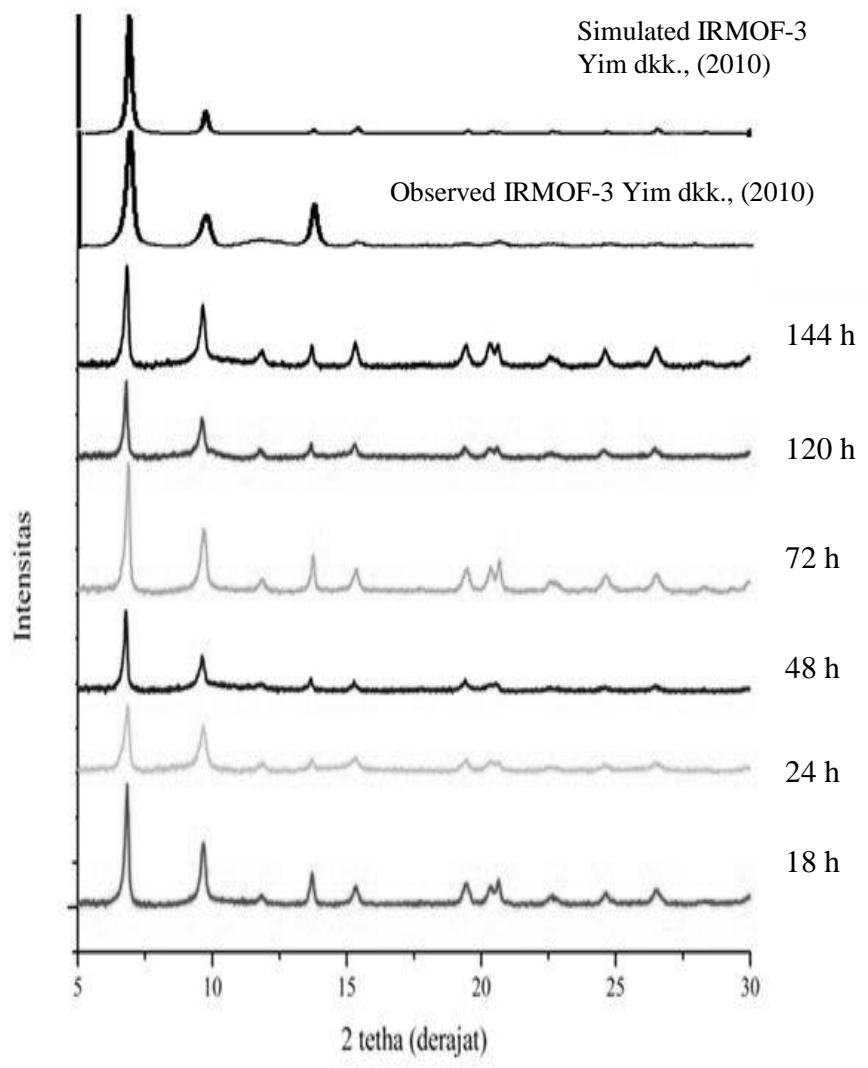



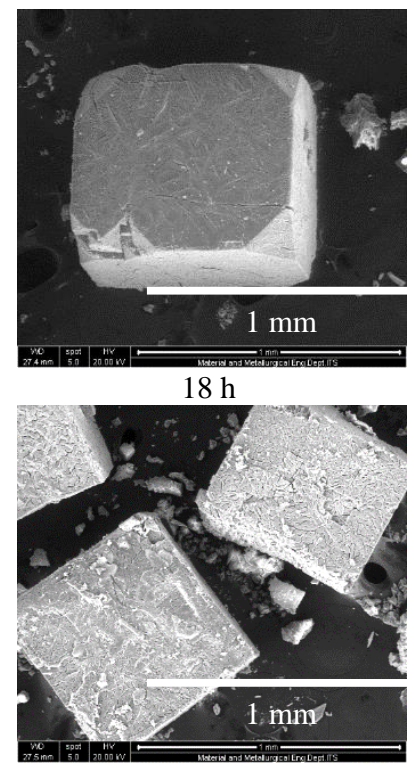

$48 \mathrm{~h}$

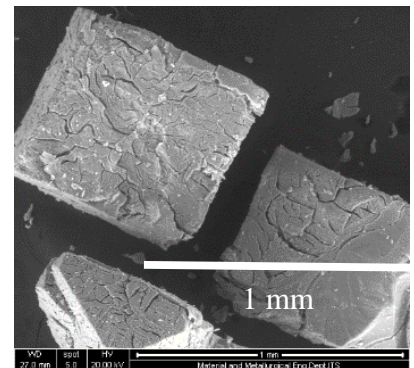

$120 \mathrm{~h}$

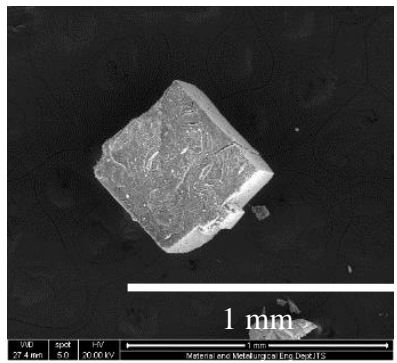

$24 \mathrm{~h}$

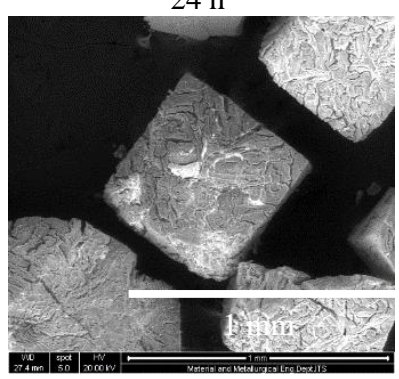

$72 \mathrm{~h}$

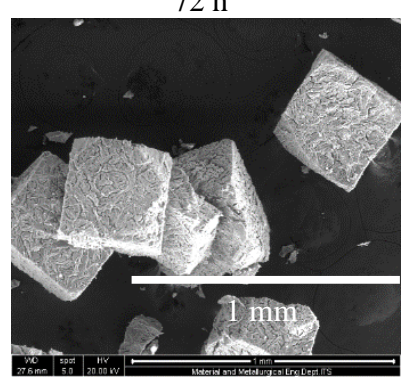

$144 \mathrm{~h}$

Figure 3. SEM micrograph of IRMOF-3 at $18 \mathrm{~h}(\mathrm{~A}), 24 \mathrm{~h}(\mathrm{~B}), 48 \mathrm{~h}(\mathrm{C})$, $72 \mathrm{~h}(\mathrm{D}), 120 \mathrm{~h}(\mathrm{E})$, dan $144 \mathrm{~h}(\mathrm{~F})$

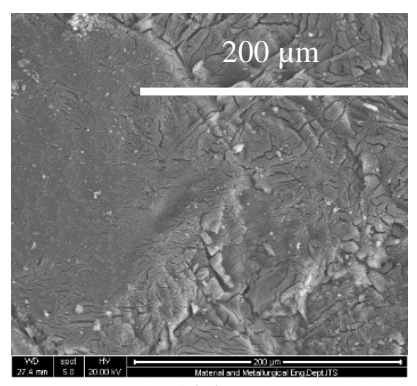

$18 \mathrm{~h}$

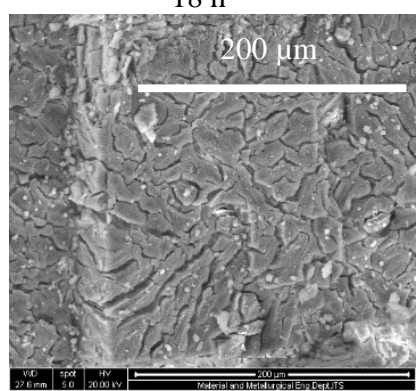

$48 \mathrm{~h}$

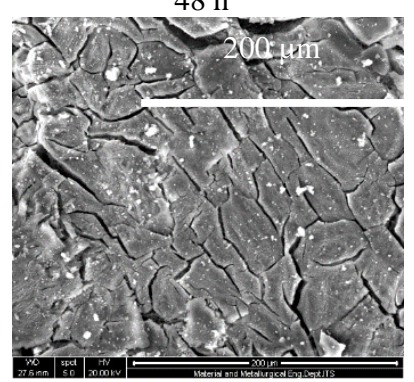

$120 \mathrm{~h}$

Figure 3. SEM micrograph of IRMOF-3 at $18 \mathrm{~h}(\mathrm{~A}), 24 \mathrm{~h}(\mathrm{~B}), 48 \mathrm{~h}(\mathrm{C})$ $72 \mathrm{~h}(\mathrm{D}), 120 \mathrm{~h}(\mathrm{E})$, dan $144 \mathrm{~h}(\mathrm{~F})$
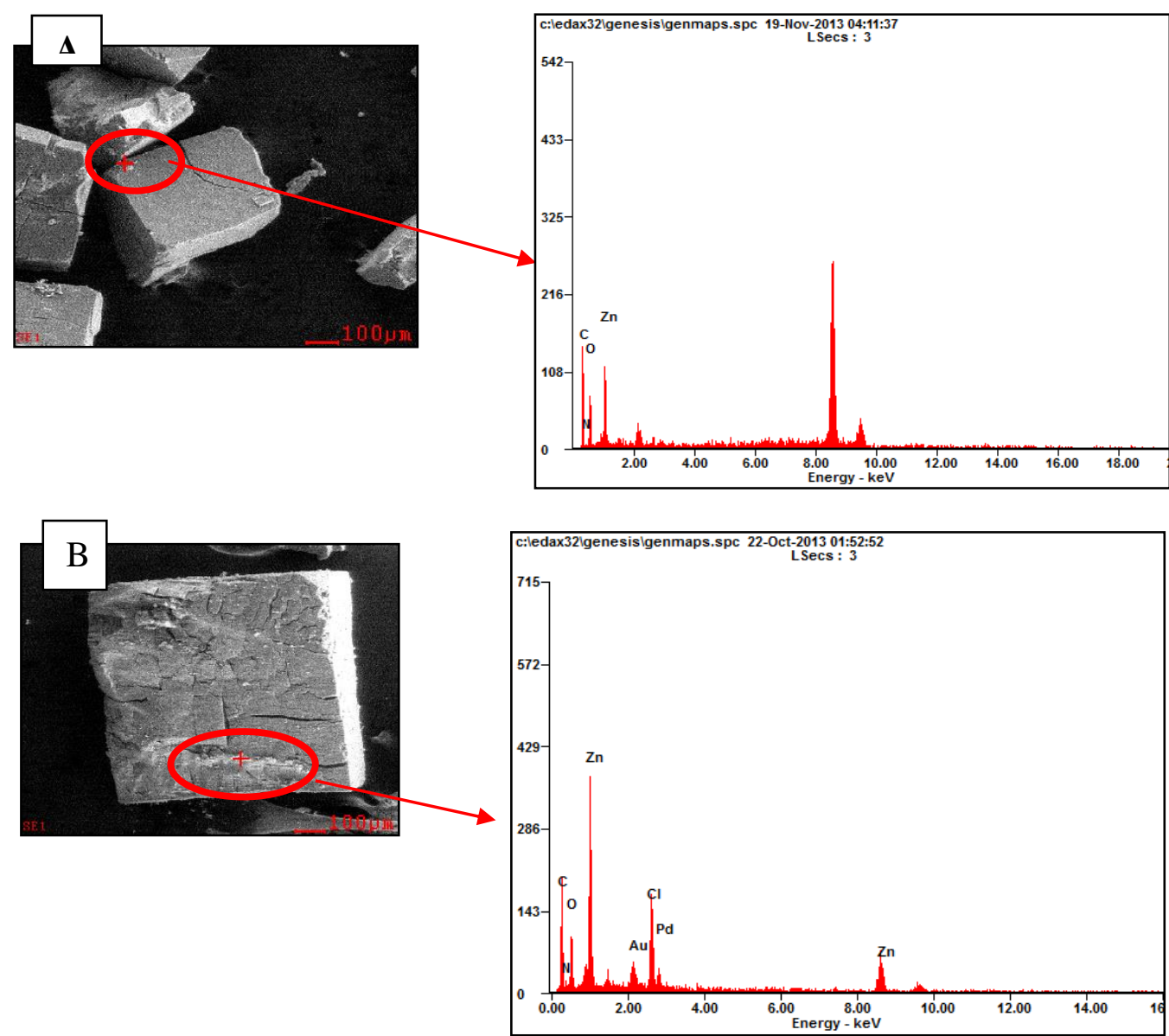


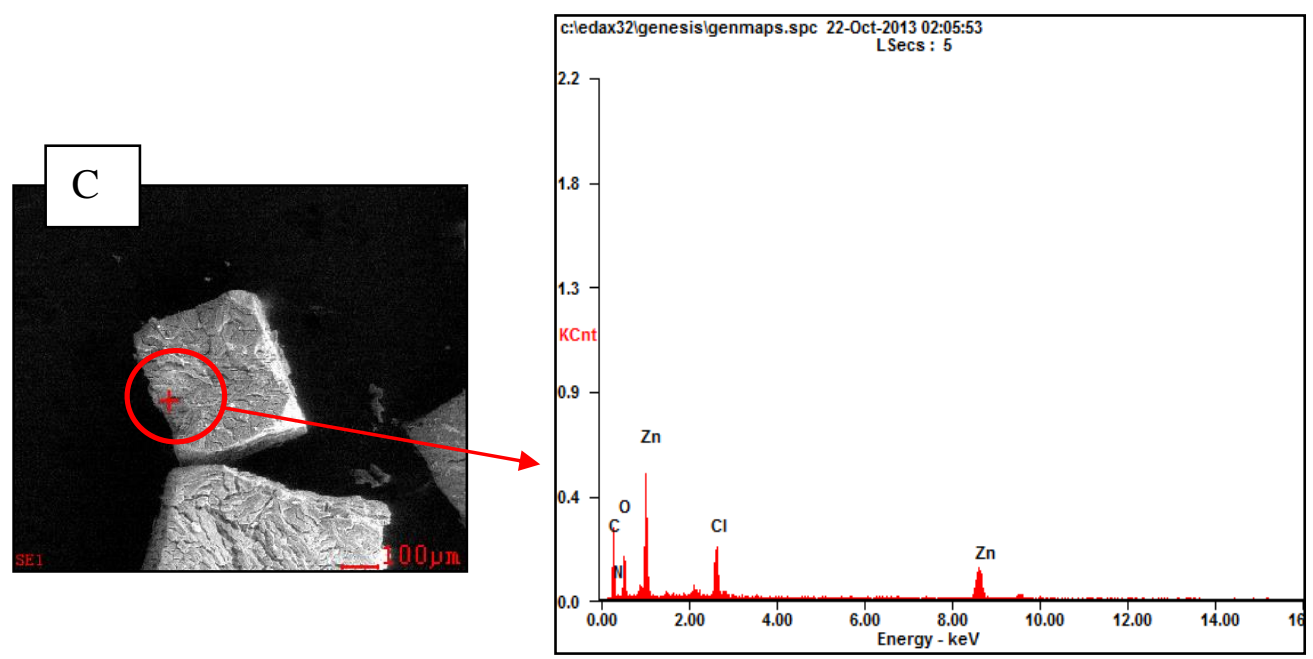

Figure 4. SEM-EDX of crystal IRMOF-3 at 24, 48 and $72 \mathrm{~h}$

TABLE 3.

PERCENT ELEMENT IN IRMOF-3 CRYSTAL

\begin{tabular}{ccccc}
\hline \hline & & & \multicolumn{2}{c}{ Wt\% } \\
No. & Element & $\begin{array}{c}\text { IRMOF-3 } \\
24 \mathrm{~h}\end{array}$ & $\begin{array}{c}\text { IRMOF-3 } \\
48 \mathrm{~h}\end{array}$ & $\begin{array}{c}\text { IRMOF-3 } \\
72 \mathrm{~h}\end{array}$ \\
\hline 1. & $\mathrm{CK}$ & 44.24 & 41,19 & 43.78 \\
2. & $\mathrm{NK}$ & 05.00 & 06,45 & 05.28 \\
3. & $\mathrm{OK}$ & 26.07 & 22,55 & 22.91 \\
4. & $\mathrm{ZnK}$ & 24.69 & 15,07 & 21.44 \\
\hline \hline
\end{tabular}

